\title{
hTERT C250T promoter mutation and telomere length as a molecular markers of cancer progression in patients with head and neck cancer
}

\author{
WOJCIECH BARCZAK ${ }^{1,2 *}$, WIKTORIA M. SUCHORSKA ${ }^{2,3^{*}}$, AGNIESZKA SOBECKA ${ }^{1,2}$, \\ KAROLINA BEDNAROWICZ ${ }^{2,4}$, PIOTR MACHCZYNSKI ${ }^{1}$, PAWEL GOLUSINSKI ${ }^{1}$, \\ BLAZEJ RUBIS ${ }^{5}$, MICHAL M. MASTERNAK ${ }^{1,6}$ and WOJCIECH GOLUSINSKI ${ }^{1}$
}

\begin{abstract}
${ }^{1}$ Department of Head and Neck Surgery, Poznan University of Medical Sciences; ${ }^{2}$ Radiobiology Lab, The Greater Poland Cancer Centre; ${ }^{3}$ Department of Electroradiology, Poznan University of Medical Sciences, Garbary, Poznan, Greater Poland Voivodeship 61-866; ${ }^{4}$ Department of Reproductive Biology and Stem Cells, Institute of Human Genetics, Polish Academy of Sciences, Strzeszynska, Poznan, Greater Poland Voivodeship 60-479; ${ }^{5}$ Department of Clinical Chemistry and Molecular Diagnostics, Poznan University of Medical Sciences, Przybyszewskiego, Poznan, Greater Poland Voivodeship 60-355, Poland; ${ }^{6}$ University of Central Florida, Burnett School of Biomedical Sciences, College of Medicine, Orlando, FL 32827, USA
\end{abstract}

Received August 24, 2016; Accepted February 27, 2017

DOI: $10.3892 / \mathrm{mmr} .2017 .6590$

\begin{abstract}
Squamous cell carcinoma of the head and neck (HNSCC) is the sixth leading cause of cancer worldwide, representing over half a million incidents every year. Cancer cells, including HNSCC, are characterized by increased telomerase activity. This enzymatic complex is active in $~ 90 \%$ of all cancer types and is responsible for the lengthening of telomeres. Highly recurrent point mutations in the human telomerase reverse transcriptase (hTERT) promoter have recently been reported in a number of human neoplasms. The aim of the present study was to analyze the prevalence of the hTERT promoter C250T mutation and telomere length in the blood leukocytes of 61 patients with HNSCC and 49 healthy individuals. Quantitative polymerase chain reaction identified the hTERT promoter mutation in 36\% of patients with HNSCC. To the best of our knowledge this is first report indicating the presence of shorter telomeres in early stage tumors. In addition, the results suggest that the C250T hTERT promoter mutation and telomere length assessment may serve as important molecular markers of HNSCC progression.
\end{abstract}

Correspondence to: Mr. Wojciech Barczak, Department of Head and Neck Surgery, Poznan University of Medical Sciences, The Greater Poland Cancer Centre, Garbary, Garbary 15 Street, Poznan, Greater Poland Voivodeship 61-866, Poland

E-mail: wojciech.barczak@wco.pl

*Contributed equally

Key words: head and neck cancer, telomerase reverse transcriptase promoter mutation, telomere length, molecular markers

\section{Introduction}

Head and neck squamous cell carcinoma (HNSCC) is the sixth most common cancer worldwide, representing over half a million incidents every year $(1,2)$. Epidemiological studies have revealed an association between exposure to carcinogens (such as tobacco and alcohol), infection by oncogenic human papillomavirus types 16 and 18 (HPV16 and HPV18, respectively) and the increased risk of HNSCC development (3). Currently, the treatment of HNSCC consists of surgery followed by postoperative chemo- and/or radiotherapy $(4,5)$. However, the 5-year mortality rate of patients with HNSCC has not improved, despite the advanced treatment methods (6).

Cancer cells, including HNSCC, are characterized by increased telomerase activity. This enzymatic complex is active in $~ 80$ to $90 \%$ of all cancer types and is responsible for the lengthening of telomeres (7). Telomeres, as highly specialized nucleoproteins located at the end of chromosomes, provide genomic stability, integrity and immortalization of cells. The human telomeric sequence is composed of hexamer repeats (5'-TTAGGG-3') at the 3 ' end strand. Human telomeres have $\sim 500$ to 2,000 copies of hexamer repetitions, giving rise to 3,000 to 12,000 base pairs (8-11).

Telomerase activity is controlled by a number of mechanisms, including alternative splicing, posttranslational modifications and activating or inhibiting factors. Thus, the transcriptional regulation of the human telomerase reverse transcriptase (hTERT) gene, followed by the regulation of telomerase activity, is crucial (12). Highly recurrent point mutations in the hTERT promoter have been reported in a number of human malignancies such as melanoma and glioma. This mutation is located at $-146 \mathrm{bp}$ (C250T) from the ATG start site of the hTERT gene (13). This mutation generates de novo E-Twenty six (ETS)/ternary complex factors 
transcription-binding sites. This process results in increased hTERT expression and it has been proposed as a novel mechanism of activating telomerase in malignant cells $(13,14)$. A previous study investigating telomerase and telomere length, indicated that their regulation may be controlled by factors released during carcinogenesis such as hormones and cytokines, including adiponectin and interleukin (IL)-6 (15). These changes, if they are present in the human organism even in the local area (cancer initiation at a single cell level), may be reflected by alterations in the whole organism including leukocytes (16).

Considering the results of our previous studies (16), it was hypothesized that short telomere length and a hTERT mutation in leukocytes may be evaluated as markers of prognosis and tumors presenting at a very early stage of carcinogenesis. Thus, telomere length measurements in leukocytes may be an effective, non-invasive method for the predictive assessment of carcinogenesis. Therefore, the present study focused on the potential application of telomere length and hTERT mutation analysis as prognostic parameters of cancer progression in HNSCC.

\section{Materials and methods}

Patients. The study group consisted of 46 patients (31 males and 15 females; age 21-88 years, median $=67$, mean $=63$ ) who were histologically diagnosed with HNSCC at various stages, and different anatomical sites based on World Health Organization criteria (Table I). All patients were recruited between April 2015 and June 2016 from the Department of Head and Neck Surgery at the Poznan University of Medical Sciences and The Greater Poland Cancer Centre in Poznan, Poland. The control group comprised of 49 healthy blood donors from Poznan Regional Blood Center. The patients were Caucasians and the majority were from the same region of Poland (Greater Poland). The study protocol was approved by the Ethics Committee of the Poznan University of Medical Sciences (Decision no. 446/15) and written informed consent was provided by the participating individuals.

Exclusion criteria. Following the study protocol, patients with local recurrences, second primary tumors and were HPV positive were excluded from experimental groups. Patients with a previous history of chemo- or radiotherapy were also excluded.

DNA isolation. DNA was extracted from $300 \mu 1$ peripheral blood mononuclear cells (PBMCs) using a Wizard Genomic DNA Purification kit (Promega Corporation, Madison, WI, USA) according to manufacturer's protocol. DNA was then stored at $-20^{\circ} \mathrm{C}$ until analysis.

\section{Quantitative polymerase chain reaction ( $q P C R$ )}

Assessment of relative telomere length. Telomere length was assessed using 2 pairs of primers, one telomere-specific and one a single copy gene-specific (albumin) (Table II), as previously described (16-18). Briefly, specific primers with an efficiency close to $100 \%(98 \pm 2 \%)$, were used in a SYBR Green-based assay with FastStart Essential SYBR Green I Master (Roche Applied Science, Penzberg, Germany). Initial denaturation and polymerase activation (hot start) was
Table I. Characteristics of the present study's cohort.

\begin{tabular}{lc}
\hline Characteristic & Total number (n) \\
\hline Healthy individuals & 49 \\
TERT promoter status [n(\%)] & \\
T/T & $13(26.5)$ \\
C/T & $29(59.2)$ \\
C/C & $7(14.3)$ \\
Cancer patients & 61 \\
Age (years) & \\
Median & 63 \\
Mean & 62 \\
Range & 37 to 88 \\
Sex [n(\%)] & \\
Male & $49(80.3)$ \\
Female & $12(19.7)$ \\
Tumor stage (TNM classification) [n(\%)] & \\
T1 & $11(18.0)$ \\
T2 & $22(36.1)$ \\
T3 & $17(27.9)$ \\
T4 & $11(18.0)$ \\
Anatomical site (n) & \\
Mouth (including lip) & 25 \\
Voice box (larynx) & 25 \\
Nose and sinuses & 5 \\
Throat (pharynx) & 6 \\
TERT promoter status [n(\%)] & \\
T/T & $23(37.7)$ \\
C/T & $16(26.2)^{\mathrm{a}}$ \\
C/C & $22(36.1)^{\mathrm{b}}$ \\
Total study cohort (n) & 110 \\
\hline
\end{tabular}

TERT, telomerase reverse transcriptase; C/C, homozygous for wild-type $\mathrm{C}$ allele; $\mathrm{C} / \mathrm{T}$, heterozygous $\mathrm{C} / \mathrm{T}$ allele; $\mathrm{T} / \mathrm{T}$, homozygous for mutant $\mathrm{T}$ allele; ${ }^{\mathrm{a}} \mathrm{P}<0.0001$ vs. healthy group; ${ }^{\mathrm{b}} \mathrm{P}<0.0001$ vs. healthy group.

performed at $95^{\circ} \mathrm{C}$ for $10 \mathrm{~min}$. The signal was detected during 45 cycles of $95^{\circ} \mathrm{C}$ for $10 \mathrm{sec}, 61^{\circ} \mathrm{C}$ for $10 \mathrm{sec}$ and $72^{\circ} \mathrm{C}$ for $10 \mathrm{sec}$. Melting analysis $\left(65\right.$ to $95^{\circ} \mathrm{C} ; 0.2^{\circ} \mathrm{C}$ resolution) was performed at the end of the reaction to verify specificity of the product. Telomere length was assessed using a qPCR system (LC 96; Roche Diagnostics, Basel, Switzerland), and calculated using the $2^{-\Delta \Delta \mathrm{Cq}}$ method (19).

High resolution melting (HRM) mutation analysis. To identify the C250T hTERT promoter mutation, HRM analysis using specific primers was performed (sequences listed in Table II). The reaction was optimized to $10 \mathrm{ng}$ genomic DNA, $0.8 \mu \mathrm{M}$ of each primer, $2.5 \mathrm{mM}$ magnesium chloride and $5 \mu 1$ of LightCycler 480 High Resolution Melting Master Mix (2X; Roche Applied Science, Penzberg, Germany), in a total volume of $10 \mu 1$. The initial denaturation and polymerase activation (hot start) was performed at $95^{\circ} \mathrm{C}$ for $10 \mathrm{~min}$. The signal was detected during another 45 cycles of $95^{\circ} \mathrm{C}$ for 
Table II. Primer sequences for quantitative polymerase chain reaction.

$\begin{array}{llr}\text { ALB_F } & \text { TTTGCAGATGTCAGTGAAAGAGA } & 69 \\ \text { ALB_R } & \text { TGGGGAGGCTATAGAAAATAAGG } & \\ \text { Telo_F } & \text { ACACTAAGGTTTGGGTTTGGGTTTGGGTTTGGGTTAGTGT } & 91 \\ \text { Telo_R } & \text { GTTAGGTATCCCTATCCCTATCCCTATCCCTATCCCTAACA } & \\ \text { TERT_HRM_F } & \text { CTGCCCCTTCACCTTCCAG } & 159 \\ \text { TERT_HRM_R } & \text { AGCGCTGCCTGAAACTCG } & \end{array}$

ALB, albumin; Telo, telomere; TERT, telomerase reverse transcriptase; HRM, high resolution melting; F, forward; R, reverse.

$10 \mathrm{sec}, 57^{\circ} \mathrm{C}$ for $15 \mathrm{sec}$ and $72^{\circ} \mathrm{C}$ for $10 \mathrm{sec}$. HRM analysis ( 65 to $95^{\circ} \mathrm{C} ; 0.07^{\circ} \mathrm{C}$ resolution) at the end of the reaction was performed to identify different variants of the hTERT mutation. The HRM analysis was performed using a qPCR system (LC 96; Roche Diagnostics, Basel Switzerland).

Sequencing. A total of $10 \%$ of samples were verified by sequencing using the BigDye v3.1 kit according to the manufacturer's instructions (Applied Biosystems; Thermo Fisher Scientific, Inc., Waltham, MA, USA) and separation by ethanol extraction using the ABI Prism 3130XL (Applied Biosystems; Thermo Fisher Scientific, Inc.) in a $36 \mathrm{~cm}$ capillary and a POP7 polymer.

Statistical analysis. Statistical analysis was performed using Student's t-test, two-way analysis of variance, $\chi^{2}$ and Fisher's exact tests, which were calculated using GraphPad Prism version 5 (GraphPad Software, Inc., La Jolla, CA, USA). Data is presented as the mean \pm standard error of the mean. $\mathrm{P}<0.05$ was considered to indicate a statistically significant difference.

\section{Results}

Telomere length assessment. The average relative telomere length (AU) in the cancer and control groups (14.06 \pm 2.109 and $19.06 \pm 1.801$, respectively) was evaluated, and no significant difference was observed $(\mathrm{P}=0.787$; Fig. 1A). Telomere length in leukocytes in relation to tumor invasion according to the TNM Classification of Malignant Tumors (TNM) was also determined. Telomeres in leukocytes from individuals with T2 HNSCC cancer were significantly shorter when compared with those of healthy individuals $(6.329 \pm 1.864$ and $19.06 \pm 1.801$, respectively; $\mathrm{P}=0.0001$; Fig. $1 \mathrm{~B})$. There was also a significant difference in telomere length between $\mathrm{T} 2$ and T3 patients $(6.329 \pm 1.864$ and $16.94 \pm 3.301$, respectively; $\mathrm{P}=0.0063$; Fig. 1B), and T2 and T4 patients $(6.329 \pm 1.864$ and $26.3 \pm 7.615$, respectively; $\mathrm{P}=0.0028$; Fig. $1 \mathrm{~B})$. In addition, there was trend towards shortened telomeres in the first two tumor invasion stages $(\mathrm{P}=0.0003$; Fig. 1B).

The analysis of telomere lengths in different anatomical sites of patients with HNSCC demonstrated that there was a significant difference between healthy individuals and patients affected by mouth cancer $(19.06 \pm 1.801$ and $10.76 \pm 2.365$, respectively; $\mathrm{P}=0.0083$; Fig. 1C) and pharyngeal cancer $(19.06 \pm 1.801$ and $3.985 \pm 1.03$, respectively; $P=0.0063$; Fig. 1C).

TERT promoter C250T mutation assessment-TNM classification. The hTERT promoter mutation was identified in $36 \%$ of the patients with HNSCC, while $27 \%$ of healthy individuals also carried the mutation (Table I and Fig. 2A). However, the heterozygous variant was observed in $26 \%$ of the HNSCC group and 59\% of the control group $(\mathrm{P}<0.0001$; Table I and Fig. 2A). Notably, when compared with the control group (14\%), $38 \%$ of the cancer types had the C wild-type allele ( $\mathrm{P} \leq 0.0001$; Table I and Fig. 2A).

The analysis of the C250T mutations indicated that there is a significant association between the frequency of the homozygous mutation and the grade of the tumor $(\mathrm{T} 1=27 \%$; $\mathrm{T} 2=36 \%$; T3=35\%; T4=46\%; P $\leq 0.0001$; Fig. 2B).

An opposite trend was identified in the case of the wild-type allele as there was a decreasing frequency of the wild type $\mathrm{C}$ allele with increasing tumor advancement $(\mathrm{P} \leq 0.0001)$, and a significantly higher frequency of the wild type allele in $\mathrm{T} 1=55 \%, \mathrm{~T} 2=36 \%, \mathrm{~T} 3=35 \%$ and $\mathrm{T} 4=27 \%$ when compared with the control group (14\%; $\mathrm{P} \leq 0.0001$; Fig. $2 \mathrm{~B})$.

TERT promoter C250T mutation assessment-anatomical sites. The C250T mutation was identified in $40 \%$ of patients with mouth cancer $(\mathrm{P}=0.001$; Fig. $2 \mathrm{C})$ and in $20 \%$ of patients with nose and sinuses cancer $(\mathrm{P}=0.0018$; Fig. $2 \mathrm{C})$, compared with $27 \%$ of healthy individuals. In addition, a nonsignificant trend for increased incidence of the C250T mutation was observed in pharyngeal cancer $(50 \%)$ and laryngeal cancer (32\%) compared with healthy individuals (Fig. 2C).

Differences in $\mathrm{C}$ allele status were also revealed. In the control group, $14 \%$ of samples contained the wild type homozygous allele (Fig. 2C); by comparison, 44\% of mouth cancer $(\mathrm{P}=0.001), 80 \%$ of nose and sinuses cancer $(\mathrm{P}=0.0018)$, $33 \%$ of pharyngeal cancer and $24 \%$ of laryngeal cancer were homozygous for the wild type allele (Fig. 2C).

Comparisons between C250T variant and telomere length revealed no significant differences between patients with HNSCC and the healthy control group (Fig. 3A). However, it was revealed that telomeres were significantly shorter in patients with hTERT-wild and heterozygous mouth cancer than in the control group $(\mathrm{P}=0.0401$ and $\mathrm{P}=0.0252$, respectively; Fig. 3B). Significantly shorter telomeres were also 


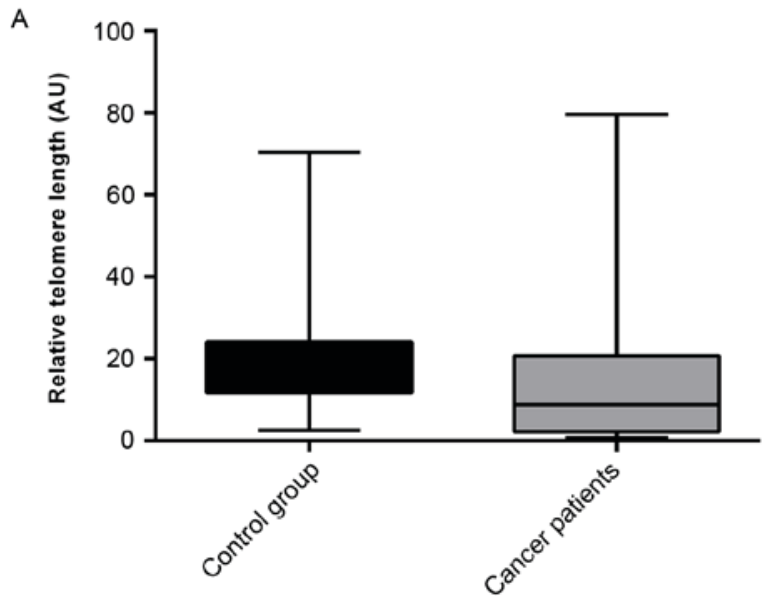

B
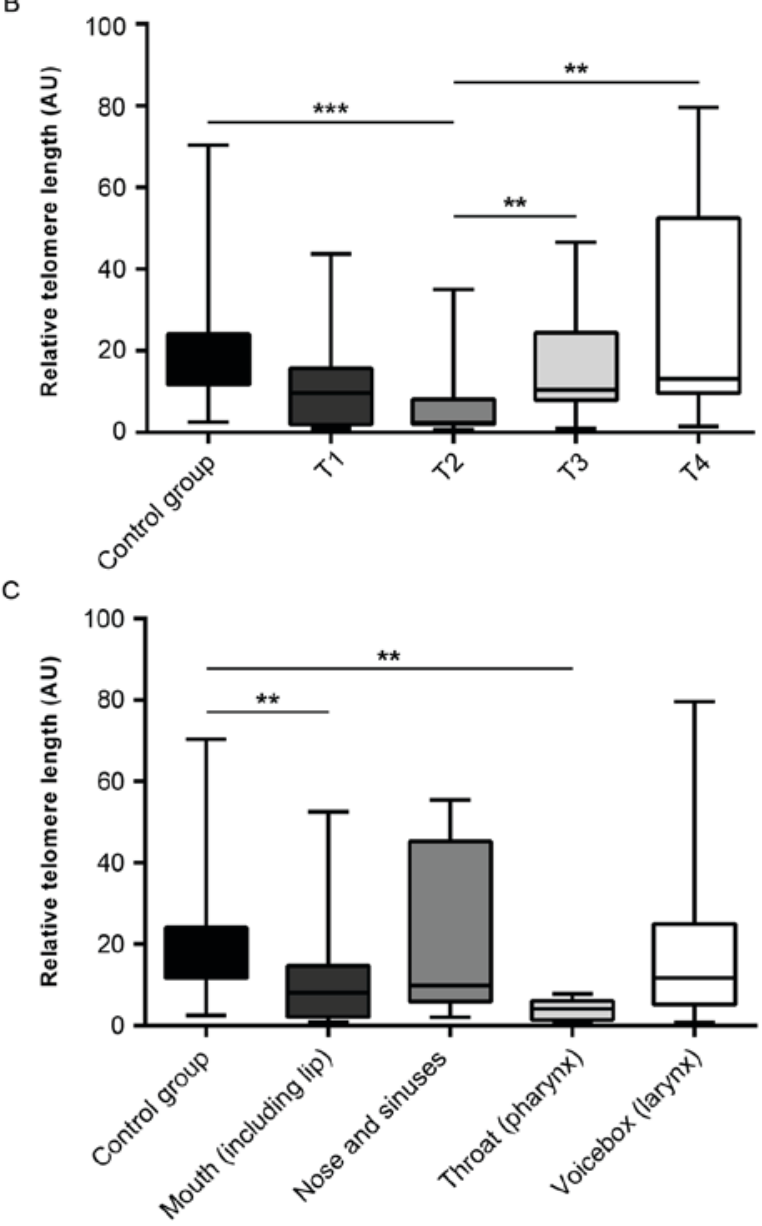

Figure 1. Quantitative analysis of relative telomere length (AU) in peripheral blood leucocytes. (A) Relative telomere length in the study (patients with HNSCC) and control (healthy individuals) groups. (B) Comparisons of relative telomere length in peripheral blood leucocytes in relation to TNM classification. (C) Comparisons of relative telomere length in peripheral blood leucocytes in relation to the anatomical sites of HNSCCs. Data is presented as the mean \pm standard error of the mean. ${ }^{* *} \mathrm{P}<0.01$ and ${ }^{* * * *} \mathrm{P}<0.001$, with comparisons indicated by lines. HNSCC, head and neck squamous cell carcinoma; TNM, TNM Classification of Malignant Tumors.

observed in hTERT-wild $(\mathrm{P}=0.026)$ pharyngeal cancer. The results exhibited a trend suggesting that hTERT-mutant laryngeal tumors have longer telomeres than the control group, however the differences were not significant $(28.861 \pm 8.198$ and $17.849 \pm 3.115$, respectively; $\mathrm{P}=0.195$; Fig 3B).
A

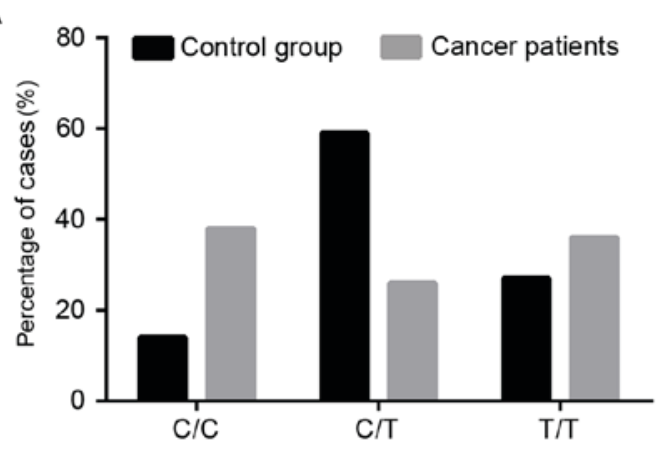

B
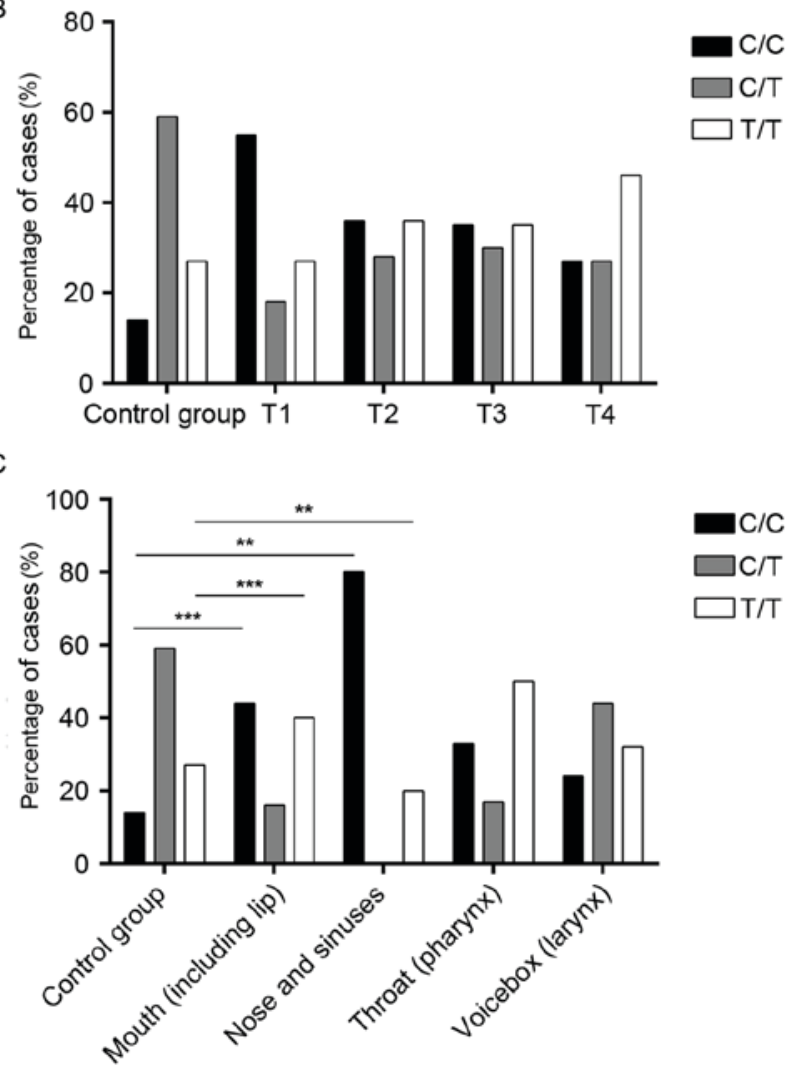

Figure 2. Frequency of C250T TERT promoter mutation in patients with HNSCC. (A) Frequency of TERT promoter mutations in the study (patients with HNSCC) and control (healthy individuals) groups. Statistical analysis of results count by $\chi^{2}$ test. $\mathrm{P} \leq 0.0001 ; \chi^{2}=25,17 ; \mathrm{df}=2$. (B) Comparisons of TERT promoter mutation frequencies in peripheral blood leucocytes in relation to TNM classification. Statistical analysis of results count by $\chi^{2}$ test $\mathrm{P} \leq 0.0001 ; \chi^{2}=63,87 ; \mathrm{df}=8$. (C) Comparisons of TERT promoter mutation frequencies in peripheral blood leucocytes in relation to the anatomical sites of HNSCCs. ${ }^{* *} \mathrm{P}<0.01$ and ${ }^{* * *} \mathrm{P}<0.001$, with comparisons indicated by lines. TERT, telomerase reverse transcriptase; HNSCC, head and neck squamous cell carcinoma; $\mathrm{C} / \mathrm{C}$, homozygous for wild-type $\mathrm{C}$ allele; $\mathrm{C} / \mathrm{T}$, heterozygous $\mathrm{C} / \mathrm{T}$ allele; $\mathrm{T} / \mathrm{T}$, homozygous for mutant $\mathrm{T}$ allele; TNM, TNM Classification of Malignant Tumors.

\section{Discussion}

In the present study, the frequency of the C250T hTERT promoter mutation in patients with HNSCC, identification of the mutation associated with telomere length and the association between cancer advancement and telomere length and mutation status was determined.

There have been a number of studies that have correlated telomere length in leukocytes and cancer risk, however only 

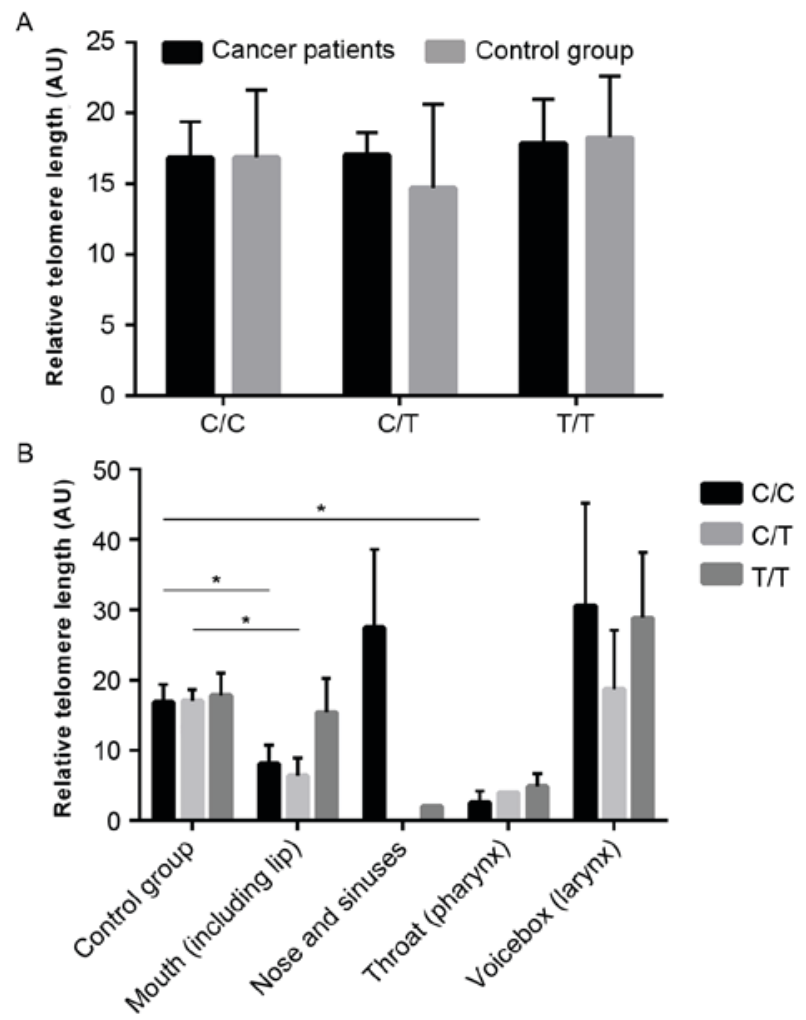

Figure 3. Assessment of relative telomere length (AU) in peripheral blood leucocytes relative to TERT promoter mutation (C250T) status. (A) Relative telomere length in the study (patients with HNSCC) and control (healthy individuals) groups. (B) Comparisons of relative telomere length in peripheral leucocytes in relation to the anatomical sites of HNSCCs. Data is presented as the mean \pm standard error of the mean. ${ }^{*} \mathrm{P}<0.05$, with comparisons indicated by lines. TERT, telomerase reverse transcriptase; HNSCC, head and neck squamous cell carcinoma; $\mathrm{C} / \mathrm{C}$, homozygous for wild-type $\mathrm{C}$ allele; $\mathrm{C} / \mathrm{T}$, heterozygous $\mathrm{C} / \mathrm{T}$ allele; $\mathrm{T} / \mathrm{T}$, homozygous for mutant $\mathrm{T}$ allele.

a few focused on head and neck cancer. Zhang et al (20) demonstrated that short telomere length in PBMCs is strongly associated with a moderately increased risk of oropharyngeal squamous cell carcinoma however, not with increased risk of oral cavity cancer. Associations between telomere length and a higher risk of HPV16-positive oropharyngeal carcinoma and tumor HPV16 status were also revealed (20). Bau et al (21) observed that short leukocyte telomere length was associated with an increased risk of developing oral premalignant lesions and precursors of oral squamous cell carcinoma. However, the opposite was revealed by Liu et al (22) who reported that relative telomere length may not be important in HNSCC carcinogenesis. A correlation between mouth cancer and pharyngeal cancer, and significantly shorter telomeres when compared to the control group was identified in the present study. Similar results were obtained by Bau et al (21) and Zhang et al (20). These studies demonstrated that short telomeres were a consistent feature of oral and pharyngeal cancer. Notably, the present study demonstrated that leukocytes from individuals with early stage T2 HNSCC tumors are characterized by shorter telomeres than leukocytes from healthy donors. The expected increase in the length of telomeres was observed in leukocytes from T3 and T4 patients when compared with T2 individuals. There is evidence that suggests that the advancement of the tumors may correlate with long telomeres and poor prognosis (23).
Increased telomere length indicates that telomere maintenance may also be important for the progression of HNSCC.

The presence of the C250T hTERT promoter mutation results in increased hTERT expression, for which a novel mechanism of activating telomerase in malignant cells has been proposed (24). Only a few studies have described the correlation between hTERT mutation status and the risk of HNSCC. In the present study, hTERT promoter mutations were identified in $36 \%$ of patients with HNSCC. Similar results have been obtained by Vinothkumar et al (25). The correlation between two hot spot mutations and the patient's clinicopathological phenotype was analyzed by the authors and a relatively high frequency of hTERT hot spot mutations in oral squamous cell carcinoma was identified. Qu et al (26) observed hTERT promoter mutations in a large group of laryngeal cancers. A strong association between these mutations and poor survival rates in patients with laryngeal cancer was demonstrated as an independently variable with respect to tumor localization or tumor invasion. An increased frequency of hTERT mutations in laryngeal and pharyngeal cancers was also established in the present study. A significantly higher frequency of the hTERT mutation was revealed in patients with mouth cancer. Notably, the opposite result was observed in nose and sinuses cancer. In addition, a significantly higher frequency of the hTERT mutation was observed in more advance tumors with increasing tendency. The results highlight the potential influence of the hTERT promoter mutation in the progression of HNSCC. Notably, trends towards the decreasing frequency of wild allele with increasing tumor advancement, and a significantly higher frequency of $\mathrm{C}$ allele in $\mathrm{T} 1, \mathrm{~T} 2$ and $\mathrm{T} 3$ tumors when compared to the control group were also described.

By contrast with the present study, the majority of previous studies in the head and neck area were focused on thyroid cancer. Vingare et al (24) demonstrated that the hTERT promoter mutations are relatively frequent in specific types of human cancers including thyroid cancer. Liu et al (27) also revealed that hTERT promoter mutations are common, particularly in follicular thyroid cancer and BRAF mutation-positive papillary thyroid cancer, and are associated with aggressive clinicopathological phenotypes. In addition, the mutation in the hTERT promoter was associated with decreased survival in patients with papillary thyroid carcinoma (28), and the hTERT promoter mutation is particularly prevalent in aggressive thyroid cancers (29). Notably, the present study revealed a trend with an increased frequency of the wild allele in mouth, nose and sinuses, pharyngeal and laryngeal cancers when compared to healthy individuals.

In addition, the results revealed significantly shorter telomeres in hTERT-wild and heterozygous mouth cancer, and in hTERT-wild pharyngeal cancer, when compared with the control group. To the best of our knowledge this association has not previously been identified in HNSCC. These results indicate that the hTERT promoter mutation may increase or decrease telomere length depending on the tumor localization and advancement of cancer.

In conclusion, the results demonstrated that the C250T hTERT promoter mutation may represent a common event during carcinogenesis in patients with HNSCC, which together with telomere length assessment may be one of the molecular markers of HNSCC progression. The present study 
also revealed an association between short telomeres and the presence of the C250T mutation, which depends on the anatomical site of cancer and tumor invasion status. However, long or short telomeres in the PBMCs of patients with HNSCC do not necessarily indicate the presence or absence of the hTERT mutation, therefore the two parameters should be considered to characterize patient status. The present study has highlighted that there should be a greater emphasis on using the length of telomeres in PBMCs as biomarkers of HNSCC. Future studies should be performed with long-term follow ups and larger study groups to determine the clinical importance of C250T hTERT promoter mutation status in the absence and/or presence of short telomeres.

\section{Acknowledgements}

The present study was supported by grants from the National Science Centre, Poland (grant no. 2015/17/N/NZ5/ 00686) and The Greater Poland Cancer Centre (grant no. 9/11/2015/PRB/WCO/21).

\section{References}

1. Thomas SM and Grandis JR: The current state of head and neck cancer gene therapy. Hum Gene Ther 20: 1565-1575, 2009.

2. Suh Y, Amelio I, Guerrero Urbano T and Tavassoli M: Clinical update on cancer: Molecular oncology of head and neck cancer. Cell Death Dis 5: e1018, 2014.

3. Hashibe M, Brennan P, Benhamou S, Castellsague X, Chen C, Curado MP, Dal Maso L, Daudt AW, Fabianova E, Fernandez L, et al: Alcohol drinking in never users of tobacco, cigarette smoking in never drinkers, and the risk of head and neck cancer: Pooled analysis in the International Head and Neck Cancer Epidemiology Consortium. J Natl Cancer Inst 99: 777-789, 2007.

4. Szeszko B, Osowiecka K, Rucińska M, Wasilewska-Teśluk E, Gliński K and Kepka L: Smoking during radiotherapy for head and neck cancer and acute mucosal reaction. Rep Pract Oncol Radiother 20: 299-304, 2015.

5. Vojtíšek R, Ferda J and Fínek J: Effectiveness of PET/CT with (18)F-fluorothymidine in the staging of patients with squamous cell head and neck carcinomas before radiotherapy. Rep Pract Oncol Radiother 20: 210-216, 2015.

6. Hashibe M, Brennan P, Chuang SC, Boccia S, Castellsague X, Chen C, Curado MP, Dal Maso L, Daudt AW, Fabianova E, et al: Interaction between tobacco and alcohol use and the risk of head and neck cancer: Pooled analysis in the international head and neck cancer epidemiology consortium. Cancer Epidemiol Biomarkers Prev 18: 541-550, 2009.

7. Chen $\mathrm{CH}$ and Chen RJ: Prevalence of telomerase activity in human cancer. J Formos Med Assoc 110: 275-289, 2011.

8. McEachern MJ, Krauskopf A and Blackburn EH: Telomeres and their control. Annu Rev Genet 34: 331-358, 2000.

9. Hug $\mathrm{N}$ and Lingner $\mathrm{J}$ : Telomere length homeostasis. Chromosoma 115: 413-425, 2006.

10. Bibby MC: An introduction to telomeres and telomerase. Mol Biotechnol 24: 295-301, 2003.

11. Suchorska WM, Augustyniak E and Łukjanow M: Genetic stability of pluripotent stem cells during anti-cancer therapies. Exp Ther Med 11: 695-702, 2016.

12. Chan S and Blackburn EH: Telomeres and telomerase. Philos Trans R Soc Lond B Biol Sci 359: 109-121, 2004.
13. Killela PJ, Reitman ZJ, Jiao Y, Bettegowda C, Agrawal N, Diaz LA Jr, Friedman AH, Friedman H, Gallia GL, Giovanella BC, et al: TERT promoter mutations occur frequently in gliomas and a subset of tumors derived from cells with low rates of self-renewal. Proc Natl Acad Sci USA 110: 6021-6026, 2013.

14. Jangard M, Zebary A, Ragnarsson-Olding B and Hansson J: TERT promoter mutations in sinonasal malignant melanoma: A study of 49 cases. Melanoma Res 25: 185-188, 2015.

15. Khan S, Shukla S, Sinha S and Meeran SM: Role of adipokines and cytokines in obesity-associated breast cancer: Therapeutic targets. Cytokine Growth Factor Rev 24: 503-513, 2013.

16. Barczak W, Rozwadowska N, Romaniuk A, Lipińska N, Lisiak N, Grodecka-Gazdecka S, Książek K and Rubiś B: Telomere length assessment in leukocytes presents potential diagnostic value in patients with breast cancer patients. Oncol Lett 11: 2305-2309, 2016.

17. Cawthon RM: Telomere length measurement by a novel monochrome multiplex quantitative PCR method. Nucleic Acids Res 37: e21, 2009.

18. Gruszecka A, Kopczyński P, Cudziło D, Lipińska N, Romaniuk A, Barczak W, Rozwadowska N, Totoń E and Rubiś B: Telomere shortening in down syndrome patients-when does it start? DNA Cell Biol 34: 412-417, 2015.

19. Livak KJ and Schmittgen TD: Analysis of relative gene expression data using real-time quantitative PCR and the 2(-Delta Delta C(T)) method. Methods 25: 402-408, 2001.

20. Zhang Y, Sturgis EM, Dahlstrom KR, Wen J, Liu H, Wei Q, Li G and Liu Z: Telomere length in peripheral blood lymphocytes contributes to the development of HPV-associated oropharyngeal carcinoma. Cancer Res 73: 5996-6003, 2013.

21. Bau DT, Lippman SM, Xu E, Gong Y, Lee JJ, Wu X and Gu J: Short telomere lengths in peripheral blood leukocytes are associated with an increased risk of oral premalignant lesion and oral squamous cell carcinoma. Cancer 119: 4277-4283, 2013.

22. Liu Z, Ma H, Wei S, Li G, Sturgis EM and Wei Q: Telomere length and TERT functional polymorphisms are not associated with risk of squamous cell carcinoma of the head and neck. Cancer Epidemiol Biomarkers Prev 20: 2642-2645, 2011.

23. Oh BK, Kim H, Park YN, Yoo JE, Choi J, Kim KS, Lee JJ and Park C: High telomerase activity and long telomeres in advanced hepatocellular carcinomas with poor prognosis. Lab Invest 88: 144-152, 2008.

24. Vinagre J, Almeida A, Pópulo H, Batista R, Lyra J, Pinto V, Coelho R, Celestino R, Prazeres H, Lima L, et al: Frequency of TERT promoter mutations in human cancers. Nat Commun 4: 2185,2013

25. Vinothkumar V, Arunkumar G, Revathidevi S, Arun K, Manikandan M, Rao AK, Rajkumar KS, Ajay C, Rajaraman R, Ramani R, et al: TERT promoter hot spot mutations are frequent in Indian cervical and oral squamous cell carcinomas. Tumour Biol 37: 7907-7913, 2016.

26. Qu Y, Dang S, Wu K, Shao Y, Yang Q, Ji M, Shi B and Hou P: TERT promoter mutations predict worse survival in laryngeal cancer patients. Int J Cancer 135: 1008-1010, 2014.

27. Liu X, Qu S, Liu R, Sheng C, Shi X, Zhu G, Murugan AK, Guan $\mathrm{H}$, Yu H, Wang Y, et al: TERT promoter mutations and their association with BRAF V600E mutation and aggressive clinicopathological characteristics of thyroid cancer. J Clin Endocrinol Metab 99: E1130-E1136, 2014.

28. George JR, Henderson YC, Williams MD, Roberts DB, Hei H, Lai SY and Clayman GL: Association of TERT promoter mutation, but not braf mutation, with increased mortality in PTC. J Clin Endocrinol Metab 100: E1550-E1159, 2015.

29. Liu X, Bishop J, Shan Y, Pai S, Liu D, Murugan AK, Sun H, El-Naggar AK and Xing M: Highly prevalent TERT promoter mutations in aggressive thyroid cancers. Endocr Relat Cancer 20: 603-610, 2013. 